Ann. Zootech., 1976, 25 (1), 79-94.

\title{
INDUCTION HORMONALE DE NAISSANCES GÉMELLAIRES : PRINCIPALES CONSÉQUENCES SUR LES PERFORMANCES ZOOTECHNIQUES
}

\author{
D. CHUPIN, N. NGUYGN HUY*, M. AZAN**, P. MALLEON \\ et R. OR'TAVANT
}

Station de Physiologie de la Reproduction. Centre de Recherches de Tours, I. N.R.A., Nouzilly, 37380 Monnaie

* I. T. E. B., 149, vue de Bercy, $7501 \%$ Paris

** C. O. O. P. E. L. S. O., Soual.

81110 Dourgne

\section{RÉSUMÉ}

Les possibilités pratiques d'utilisation de PMSG pour augmenter le taux do naissances gémel. laires ont été étudiées sur 256 vaches FFPN traites appartenant à des éleveurs du Sud-Ouest de la France. La récolte de veaux est diminués par la présence de brucellose (45.7 p. Ioo des éle vages) et l'utilisation pour l'insémination artificielle d'un taureau présentant des troubles de fécondité. Les taux de gestation après une IA et sur l'cnsemble de la campagne varient respectivement de 45,8 à 66,2 p. Ioo et cle 88,3 à 9 f, r p. Ioo. Le taux da veaux nés pour roo vaches mettant bas à la suite du traitement varie de I I 8 à 188 p. Ioo. Dans le meilleur lot (forte dose de PMSG, taureau de fécondité normale) on obtient I 29 vaux pour Ioo vaches mises en traitement.

Les veaux jumeaux naissent plus légers et plus jeunes (duré do gestation réluite) que les veaux simples mais ceci peut être limité par le choix du taureau pour l'I A et par une suralimentation des vaches en fin de gestation. Par la suite, la vitesse de croissance jusqu'à zoo jours n'apparaît pas significativement différente de celle des veaux nés simples.

Aucun effet significatif n'est observé ni sur la fertilité ultérisure des vaches ayant mis bas plusicurs veaux ni sur leur production laitière pendant ou après cette gestation multifœetale.

\section{INTRODUCTION}

Une des voies qui permettent d'améliorer la rentabilité des élevages bovins spécialisés dans la viande est l'augmentation du nombre de veaux élevés par vache. Parallèlement aux recherches sur la mortalité des veaux l'étude des possibilités 
d'augmentation de la prolificité par traitement hormonal répond à cet objectif. Après le bilan d'un essai en ferme publié par GoRdon et al. (I962) et les résultats obtenus en station expérimentale (MAULEON et al., r970) il est apparu nécessaire d'étudier les possibilités d'utilisation de cette méthode chez des petits éleveurs laitiers qui soumettraient à cette intensification une partie de leur cheptel, le plus souvent des animaux réformés pour cause de production laitière insuffisante.

\section{MATÉRIEL ET MÉTHODES}

256 vaches en lactation de race Française Frisonne Pie Noire appartenant à i 55 éleveurs du Sud-Ouest de la France (zone COOPELSO $\left.{ }^{1}{ }^{1}\right)$ : Aveyron, Haute-Garonne, Tarn) ont été traitées en deux expériences.

Tous les animaux ont reçu une injection intramusculaire de PMSG le $16^{\mathrm{e}}$ jour du premier ou deuxième cycle post-partum à la dose de I 4 oo UI (lot I : I 7 I vaches) ou I Ioo UI (lot $2: 85$ vaches). Dès le début de l'cestrus les animaux recevaient I 500 UI d'HCG (injection intraveineuse).

Les animaux du lot I revenant en ostrus i 8 à 20 jours après l'cestrus de traitement, ainsi que ceux pour lesquels cet nestrus de traitement se produisait plus de 8 jours après l'injection de PMSG, étaient traités une seconde fois selon les mêmes modalités. Les animaux du lot II au contraire n'ont reçu qu'un seul traitement : les animaux venant en cestrus plus cle Io jours après la PMSG étaient inséminés sans injection de HCG.

Lors de l'cestrus, les animaux des deux lots ont été inséminés deux fois à I 2 heures d'intervalle avec de la semence de trois taureaux de race Blonde d'Aquitaine.

Entre le $180^{e}$ et le $220^{\mathrm{e}}$ jour de la gestation, une recherche des gestations multifotales par électrocardiographie a été mise en place dans le but de suralimenter préférentiellement $(2$ à $5 \mathrm{~kg}$ d'aliment vache laitière ( $180 \mathrm{~g}$ MAI)/UF) par jour) pendant les 6 dernières semaines les vaches gestantes de plusieurs veaux. Les résultats de cette partie de l'expérience ont été publiés par ailleurs (Bosc et CHUPIN, 1975). Les parturitions étaient surveillées et réalisées par les éleveurs, ou par les vétérinaires praticiens sur appel des éleveurs, mais sans qu'une intervention systématique soit prévue.

Après la mise bas, toutes les vaches ont été traitées systématiquement (sérum antigangréneux, antibiotique) pour limiter les suites des rétentions placentaires. Les veaux ont reçu systématiquement une injection de $\gamma$-globulines colostrales.

L'année suivant le traitement, la fertilité après IA lors d'cestrus normaux a été suivie sur I 5 vaches (le reste de l'effectif étant réformé). De même, les productions laitières ont été relevées sur 3 années encadrant le traitement et comparées à celles de vaches témoins (appariées par rang de lactation et dates de vêlages dans chaque étable).

Une partie des veaux (30 mâles et 27 femelles) a été rachetée et élevée en station jusqu'à l'âge de 3 oo j (lot I).

Les 155 élevages retenus étaient indemnes de tuberculose et de fièvre aphteuse. En ce qui concerne la bruccllose, un diagnostic a été réalisé avant les traitements ( $\mathrm{I}$ Ring test et I seroagglutination pour le lot I, 3 Rings tests pour le lot II) mais il n'a pas été possible d'éliminer les élevages réagissants.

La répartition des élevages quant à leur réaction aux tests de recherche de la brucellose était la suivante :

\begin{tabular}{|c|c|c|c|c|}
\hline Réaction brucellique & Positive & Douteuse & Négative & Total \\
\hline 一 & 一 & $\cdots-$ & — & $\ldots$ \\
\hline Lot I & $\mathrm{I}_{j}$ & I 5 & 60 & 92 élevages \\
\hline Lot II & I5 & $2+$ & $2+$ & ${ }_{0} 3$ élevages \\
\hline
\end{tabular}




\section{RÉSULTATS}

\section{I. - Incidence de l'induction hormonale sur la productivité numérique des vaches}

Fécondation.

Dans le tableau I est porté le bilan des fécondations des 2 lots.

TABI,EAU I

Taux de mise bas et importance des retours en cestrus tardifs (> $90 \mathrm{j}$ )

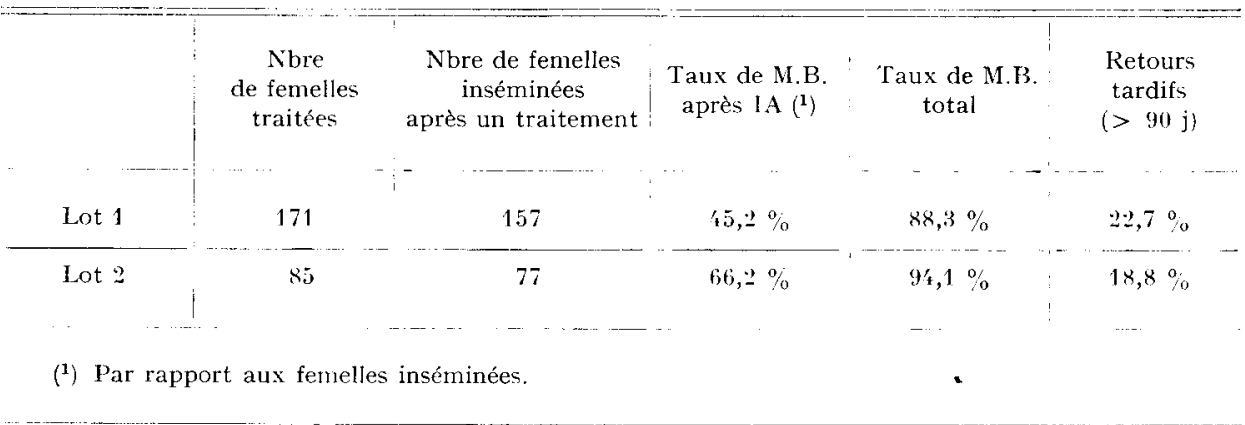

Dans le lot I (forte superovulation) I 57 des 17 I femelles traitées ont été inséminées après un traitement et $7 \mathrm{I}\left(45,2 \mathrm{p}\right.$. Ioo) ont mis bas après la $\mathrm{I}^{\mathrm{r}} \mathrm{IA}$. Le taux de nises bas cumulé est de 88,3 p. Ioo (I5I vaches) soit un taux d'animaux non fécondés de II,7 p. IOo. Le taux de retours en cestrus tardifs ( $>90$ j) après la Ire insémination est de 22,7 p. IO0. Il peut résulter d'une mortalité embryonnaire assez élevée due en partie à une trop forte superovulation et en partie à la brucellose.

Dans le lot 2 (superovulation modérée) : 77 des 85 femelles ont été inséminées après un traitement et parmi celles-ci $5 \mathrm{I}(66,2 \mathrm{p}$. Ioo) ont mis bas. Au total 80 vaches (94,I p. I0o) ont vêlé (5,9 p. Ioo des vaches sont restées vides), I8,8 p. Ioo des vaches ont eu des retours tardifs probablement attribuables en majeure partie à la brucellose, la superovulation étant modérée dans ce lot.

Mises bas.

Les bilans des vêlages des deux lots sont portés dans les tableaux 2 et 3 .

Dans le lot I on obtient 83 gestations après I ou 2 traitements dont $34(40,9$ p. IOo) (25 à terme et 9 avortements), sont des gestations multifœetales. Parmi celles-ci I7 (50 p. IOo) concernent plus de 2 foetus.

Dans le lot 2 pour $5 \mathbf{I}$ gestations consécutives au traitement on obtient Io ( I, 6 p. IOO) gestations multifoetales (7 à terme et 3 avortements) dont 2 (20 p. IOO) sont de plus de 2 foetus. 
Les animaux du lot I ont produit 107 veaux nés pour 7 I mises bas consécutives au traitement (I50,7 p. IOO) et I 74 veaux nés pour I 7 I vaches traitées (IOI,6 p. IOO).

Les animaux du lot 2 ont produit 56 veaux nés pour 48 mises bas consécutives au traitement (I I6,6 p. IO0) et 82 veaux nés pour 85 vaches traitées $(96,4$ p. I0o).

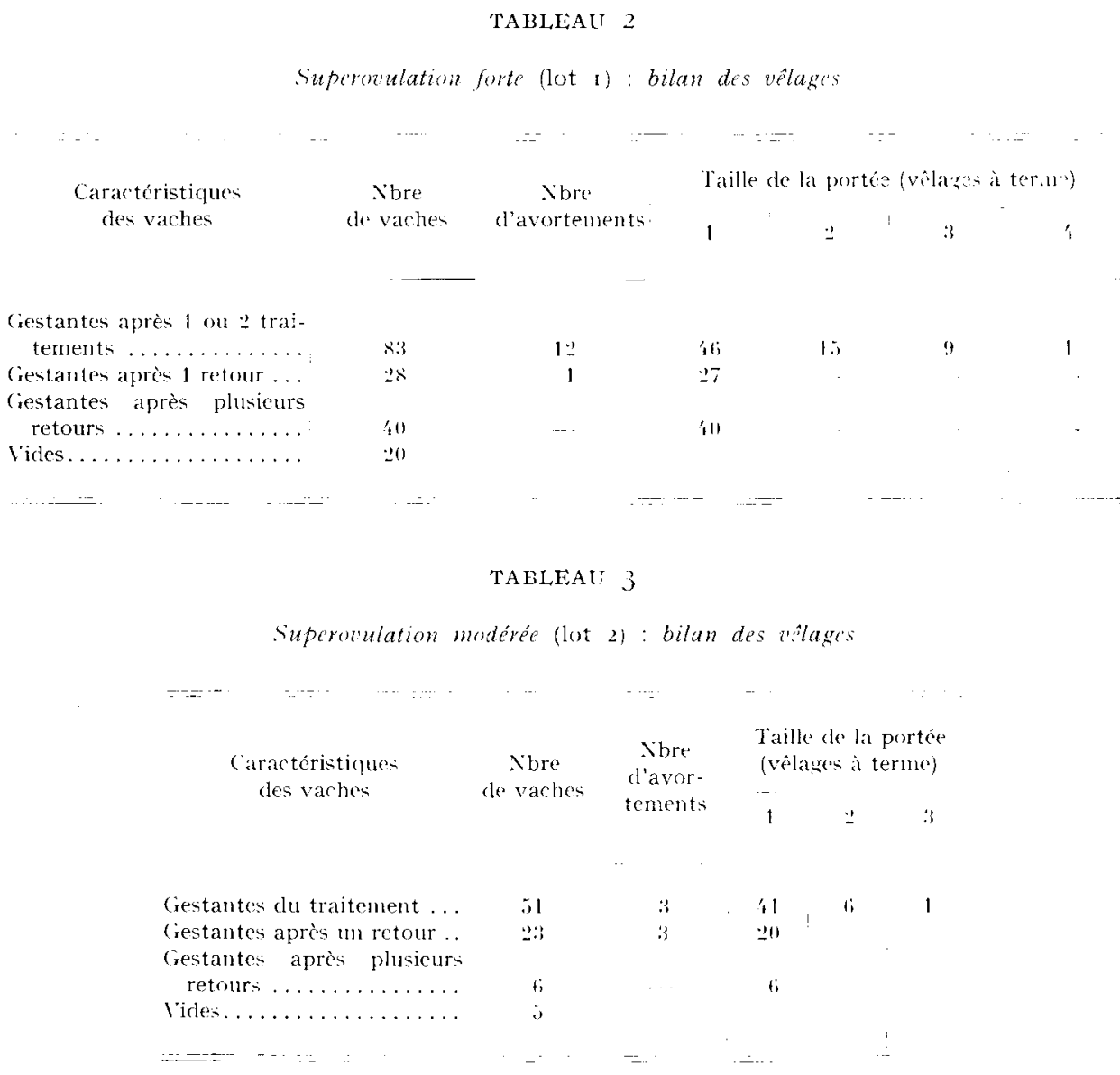

In réalité, la récolte de veaux a été très diminuée par suite de l'existence d'avortements. En effet, pour le: deux lots 3,6 p. Ion des gestations simples, i6,o p. Ioo des gestations doubles et $42, \mathbf{I}$ p. Ioo des gestations triples ou quadruples se terminent par un avortement (gestation d'une durée inférieure à $256 \mathrm{j}$ ). Cela représente $27,2 \mathrm{p}$. IoO d'avortements pour l'ensemble des gestations multifœtales et 8,2 p. I oo pour l'ensemble des animaux. On constate de plus, que ces avortements sont en relation avec les taureaux utilisés pour l'insémination artificielle.

\section{Effet Taureau.}

Effectivement, les tableaux 4 et 5 indiquent un effet du taureau utilisé à l'insémination sur le taux de conception, la répartition des retours en cestrus, le taux 
d'avortements, la prolificité et la mortalité des veaux à la naissance. Trois taureaux ont été utilisés (deux pour chaque lot). Les taux de conception varient de 46,4 à 68,8 p. Ioo entre le taureau le plus défavorable et le meilleur.

\section{TABLEAU 4}

Influence du taureau utilisé pour l'insémination artificielle sur le bilan des fécondations

\begin{tabular}{|c|c|c|c|c|c|}
\hline & \multirow{2}{*}{ Mâle } & \multirow{2}{*}{$\begin{array}{c}\text { Nbre de femelles } \\
\text { inséminées }\end{array}$} & \multirow{2}{*}{$\begin{array}{c}\text { Gestantes } \\
\text { après le traitement } \\
(\%)\end{array}$} & \multicolumn{2}{|c|}{ Retours en cestrus } \\
\hline & & & & avant $30 \mathrm{j}$ & après $30 \mathrm{j}$ \\
\hline Lot I & $\begin{array}{l}\text { Cecil } \\
\text { Cervantès }\end{array}$ & $\begin{array}{r}114 \\
51\end{array}$ & $\begin{array}{l}46,4 \\
51,0\end{array}$ & $\begin{array}{r}7,0 \\
11,7\end{array}$ & $\begin{array}{l}46,6 \\
37,3\end{array}$ \\
\hline Lot II & $\begin{array}{l}\text { Cervantès } \\
\text { Clairon }\end{array}$ & $\begin{array}{l}37 \\
45\end{array}$ & $\begin{array}{l}59,4 \\
68,8\end{array}$ & $\begin{array}{r}18,9 \\
4, t^{\prime}\end{array}$ & $\begin{array}{l}21,6 \\
26,8\end{array}$ \\
\hline Lot $\mathrm{I} / \mathrm{II}$ & Divers & 9 & 66,6 & 一 & 33,3 \\
\hline
\end{tabular}

TABLEAU 5

Infuence du taureau utilisé pour l'insémination artificielle sur le bilan des vêlages

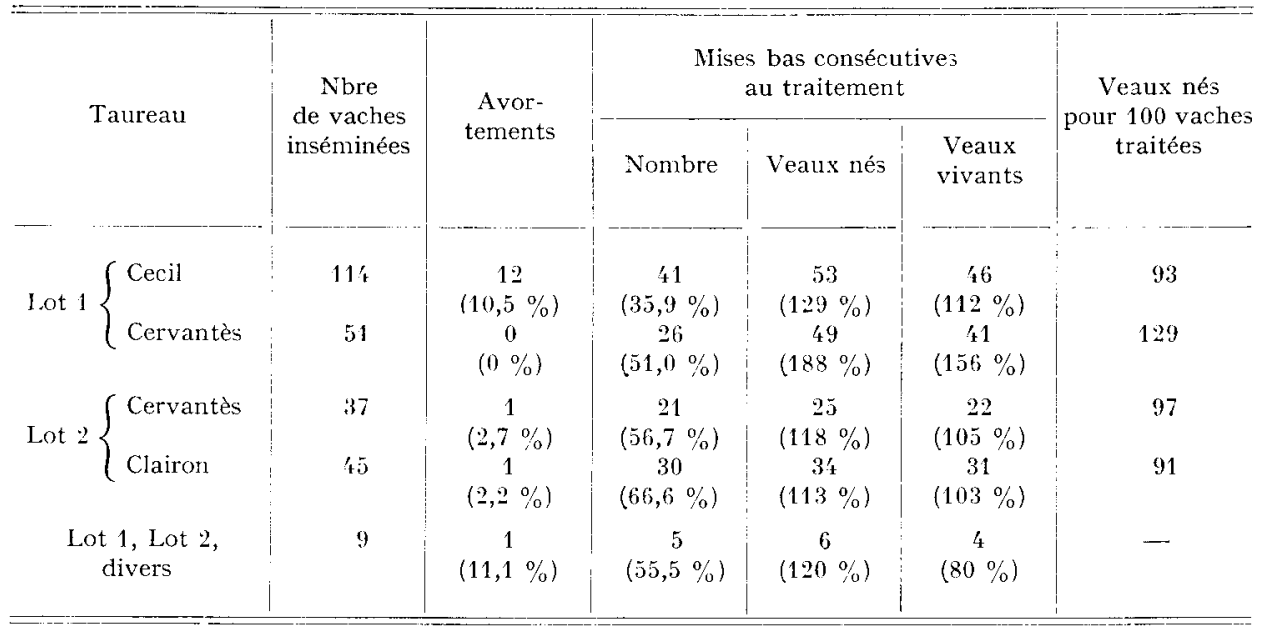

Le taureau Cécil, outre son faible taux de conception, présente un taux élevé d'avortements (IO,5 p. IOO). Les $4 \mathrm{I}$ mises bas consécutives au traitement obtenues avec ce taureau donnent 53 veaux nés ( $129 \mathrm{p}$. Ioo) et 46 veaux vivants (I 2 p. IOO).

Dans ce même lot I, donc avec la même dose de PMSG, sur les femelles inséminées avec la semence de Cervantès on n'observe aucun avortement et les 26 mises 
bas consécutives au traitement donnent 49 veaux nés (188 p. Ioo) et +I reaux vivants ( I 56 p. I0o). Il n'y a pas de différence entre taureaux dans les taux de mortinatalité.

Dans le lot 2, le taux de superovulation est trop faible pour permettre une discrimination entre taureaux sur ces critères.

\section{II. -_ Incidence de la gémellité sur le's performance's des réanx}

Wortalité des reaux et taill' de la portée.

Le tableau 6 indique le taux de mortinatalité en fonction de la taille de la portée. Nous avons regroupé dans ce tableau les mises bas après I ou 2 traitements des: deux lots, quel que soit le taureau utilisé. Le taux de mortalité augmente avec la taille de la portée passant de 2,I p. IoO pour les naissances simples à 23,8 p. Ioo pour les doubles et $32,5 \mathrm{p}$. Ioo pour les triples et quadruples. La taille de la portée restante est de 0,98 pour les naissances simples, $\mathbf{1}, 52$ pour les doubles, 2,09 pour les triples et quadruples.

\section{TABLIAU 6}

Worlalité des écaux à la naissance en fonction de la taille de la portée

\begin{tabular}{|c|c|c|c|}
\hline$\cdots$ & & $\ldots$ & $:=$ \\
\hline $\begin{array}{l}\text { Taille } \\
\text { le la portét }\end{array}$ & $\begin{array}{l}\text { Nombre } \\
\text { de veaux nés }\end{array}$ & & $\begin{array}{l}\text { Taux } \\
\text { nortalite } \\
\left({ }_{0}\right)\end{array}$ \\
\hline
\end{tabular}

$\begin{array}{cccc}1 & 31 & 2,1 & 11,98 \\ 2 & 3-2 & 23,8 & 1,22 \\ 3 \text { et } 1 & 34 & 38,5 & 2,09\end{array}$

TABI.EAU 7

Durée de gestation an fonction de la taille de la portés

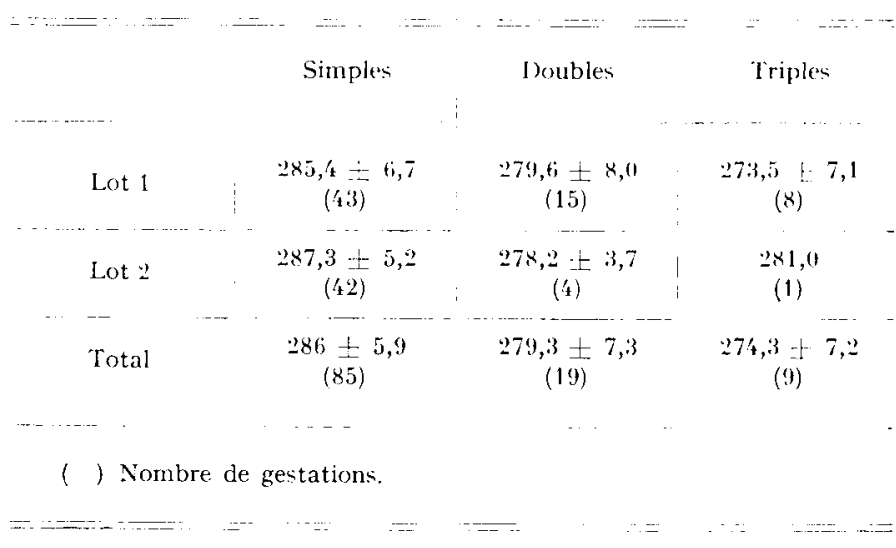


Poids de naissance des i'caux et duréc de gestation.

Les durées de gestation en fonction de la taille de la portée sont notées dans le tableau 7 . Filles sont de $286,3 \pm 5,9$ jours pour les simples, $279,3 \pm 7,3$ jours pour les doubles et $274,3 \pm 7,2$ jours pour les triples. La gestation de quadruples du premier lot a duré 280 jours.

Les poids des veaux à la naissance en fonction de la taille de la portée sont notés dans le tableau 8 . Ils sont en moyenne de $42,4 \pm 6,4 \mathrm{~kg}$ pour les simples, $3 \mathrm{I}, 3 \pm 6, \mathrm{I} \mathrm{kg}$ pour les doubles et $27,4 \pm 3,6 \mathrm{~kg}$ pour les triples. On peut noter dans

\section{TABI,EAU 8}

Poids dess veaux (kg) à la naissance. Influence du taureau

Taille de la portée

\begin{tabular}{|c|c|c|c|c|}
\hline \multirow[t]{2}{*}{ Taureau } & \multirow[b]{2}{*}{ Simples } & \multirow{2}{*}{ Joubles } & & \multirow{2}{*}{ Quadruples } \\
\hline & & & Triples & \\
\hline Cocil & $00, \frac{1}{(19)} 5,9$ & $\underset{(1 \stackrel{-1}{ \pm})}{ \pm}(i, 1$ & $\frac{3,3, x+\frac{1}{(9)}}{(9), 0}$ & $\ldots$ \\
\hline Cervantes & $\underset{(3,4)}{ \pm}(1,0$ & $32,9 \pm(2,0,3$ & $2 x, x \underset{(1 \pm)}{ \pm}: 3,6$ & $\begin{array}{l}2 x, 8 \\
(1)\end{array}$ \\
\hline Clairon & 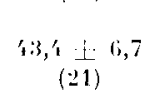 & $\begin{array}{l}35,0 \\
(2)\end{array}$ & $\begin{array}{l}=2 i, 1 ; \\
(3)\end{array}$ & -- \\
\hline Total & 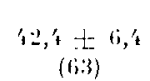 & $31,3 \underset{(36)}{ \pm}(i, 1$ & 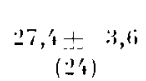 & $\begin{array}{l}2 x, x \\
(i)\end{array}$ \\
\hline
\end{tabular}

( ) Nombre de veaux.

TABLEAL 9

Influence de la suralimentation pre-partum sur la duré de gestation ot le poids des veaux à la naissance

Lot

])urce de gestation
(jours)
Poids des veaux

multiples $(\mathrm{kg})$ (doubles on triple;)

\begin{abstract}
Témoin (gestantes de plusieurs fortus non suralineutées)
\end{abstract}

Suralimente

$$
27 x \pm 2,9
$$

(x)

$$
.7,0+3, x
$$

$3: 1, \div ;, 9$

(16)

() Nombre de gestations ou de veaux. 
le lot I une différence entre les descendants de Cécil et de Cervantès ce dernier donnant des veaux multiples sensiblement plus lourds, bien que la différence soit faible au niveau des veaux simples.

Le diagnostic de gestation multifotale par électrocardiographie le $7^{\mathrm{e}}$ mois de gestation a permis de détecter $75 \mathrm{p}$. Ioo des cas de gestations multifotales. Il a été ainsi possible d'étudier l'effet de la suralimentation pré-partum sur la durée de gestation et le poids des veaux multiples (tabl. 9). On constate un allongement de 4 jours de la durée de gestation et $u n$ accroissement de $6,6 \mathrm{~kg}$ du poids de naissance des veaux multiples.

\section{Croissance des veaux.}

Le poids des veaux du lot I à la naissance, puis à I00, 200 et 300 jours est porté dans le tableau Io en fonction de la taille de la portée. Malgré un début de croissance difficile dû à un niveau nutritionnel insuffisant pendant les roo premiers jours, les veaux multiples se maintiennent à un niveau non significativement différent de celui des simples.

TABI,EAU IO

Croissance des veaux de la naissance à $300 j$

en fonction de la taille de la portźe

Lot I (mâles + femelles))

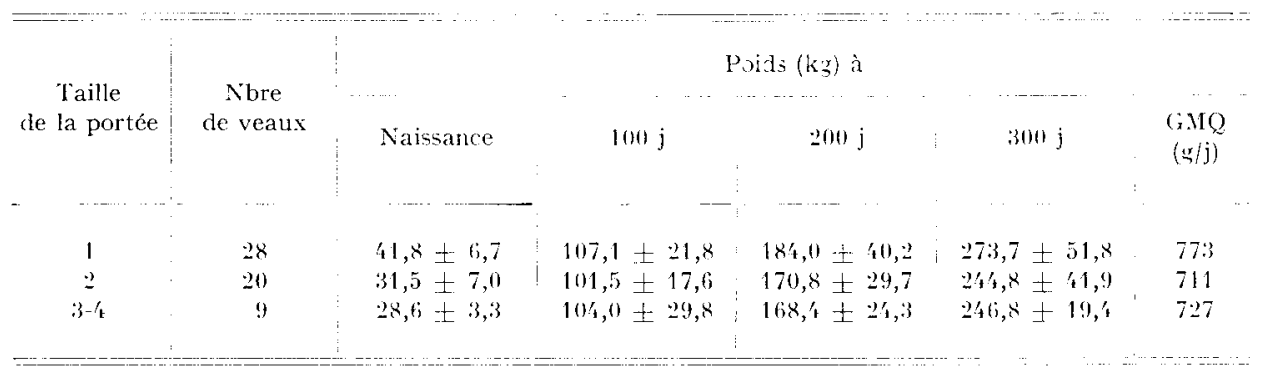

\section{III. - Incidence de l'induction hormonale' sur la performance des vaches}

Rétentions placentaires.

Les naissances multiples ont été accompagnées d'un taux élevé de rétentions placentaires (30 p. Ioo) alors qu'il a été de i I p. Ioo pour les naissances simples.

\section{Fertilité ultérieure.}

Le nombre d'inséminations nécessaires pour obtenir une fécondation l'année suivant le traitement est porté dans le tableau I en fonction du type de naissance. Les taux de conception après I IA sont de 62,3 p. Ioo après naissances simples et 72,7 p. Ioo après naissances doubles. Cette différence n'est pas significative. 


\section{TABI_EAU I I}

\section{Fortilité des vaches l'année suivant le traitement} (nombre d'lA pour obtenir une conception)

en fonction du nombre de veaux du vilage prácólent

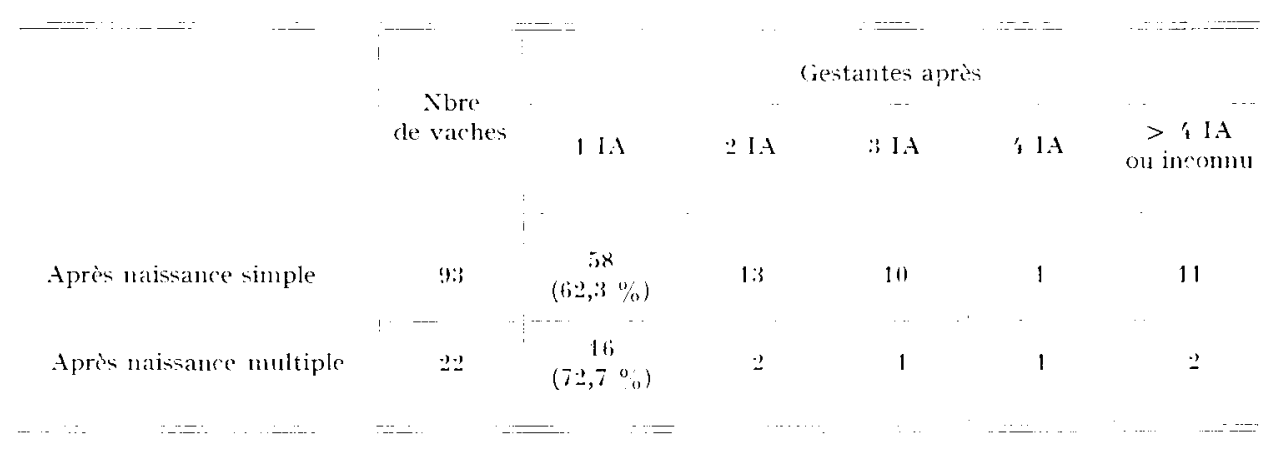

\section{Production laitièr.}

Les productions laitières des trois années avant, pendant et après le traitement sont portées dans le tableau I2. Elles sont ajustées pour une durée moyenne de 305 jours (proportion directe de la durée réelle) selon la méthode de Bowman et HENDY (I970). Les différences ne sont pas significatives; ainsi, le traitement n'a pas modifié les productions laitières.

TABI.EAU I 2

Production laitière avant, pendant et après le traitement de naissance's gémellaires (lot 1 )

(Lactation ajustée à 305 jours)

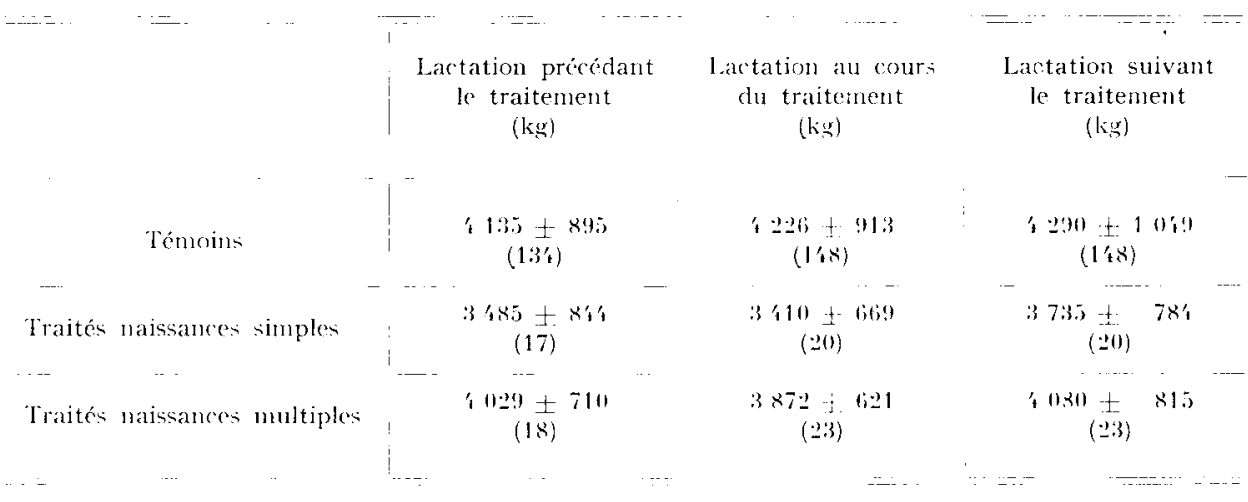

\section{DISCUSSION}

L'apport d'une technique visant à l'augmentation de la fréquence des naissances gémellaires se juge par le bilan des vêlages (fécondité) et par les conséquences zootechniques sur les mères et sur les produits. 


\section{I. - Incidence de l'induction hormonal' sur la productivité numérique}

\section{Fécondation.}

Nous avons obtenu des taux de conception (vérifiés par la mise bas) de 45,2 p. Ioo pour le lot I et 66,2 p. roo pour le lot 2 (différence significative; $\mathrm{P}<0,0 \mathrm{I}$ ). L'emploi d'une dose de PMSG plus faible sur les animaux du lot 2 s'est traduit par une superovulation très limitée accompagnée nécessairement d'une mortalité embryonnaire plus faible que pour le lot I. La fertilité de ce lot I était diminuée également du fait de 1'utilisation du taureau Cécil.

Ces taux de conception sont comparables à ceux rapportés par d'autres auteurs lors d'expériences ne comportant pas de détermination du nombre d'ovulations: 65 p. IOo (HAFEz et al., I964), 56 p. Ioo (abattage à 30-6o j) (BEI.Lows et al., I969); 44,9 p. Ioo et 59,3 p. Ioo (MAUlion et al., I970), 64, I p. Ioo (Turman et al., I97I).

Lorsqu'un contrôle du nombre d'ovulations est réalisé (palper rectal, laparotomie endoscopie), le taux de conception est plus faible $=22$ à 44 p. Ioo (ScHWARTz et SHElBy, I969) 53,4 p. IOO (MAUl.eON et al., I970) 36 p. IOO (LASTER et al., I97I) 5I p. roo (VINCENT et Milis, I972), ce qui provient vraisemblablement de la manipulation supplémentaire effectuée sur les animaux. Ceci est à rapprocher des résultats de Gordon et al. (I962) qui avec un taux de conception de 76,2 p. Ioo à 6 semaines n'obtiennent que 39, I p. Ioo de mises bas par suite d'une tentative de régulation du nombre de fœetus (éclatement de la poche amniotique).

\section{Vêlages.}

Nous avons obtenu pour les lots I et II respectivement I50 et I I6 veaux nés pour Ioo mises bas consécutives aux traitements et Ior,6 et 96,4 veaux pour IoO vaches traitées. Ce faible bilan par rapport aux vaches traitées est dî̀ à un taux élevé d'avortements que l'on peut imputer en partie à l'effet d'un taureau (lot I) et en partie à un effet de la brucellose $(34 \mathrm{p}$. roo et $62 \mathrm{p}$. Ioo respectivement des élevages sont positifs ou douteux lors des tests de recherches de la brucellose dans les lots I et 2).

Les bilans de vêlages publiés par divers auteurs ont été regroupés dans le tableau I3. Ils sont en général comparables à ceux qui sont rapportés ici exception faite de l'expérience de Turman et al. (I97I) qui utilisent la PMSG à deux moments du cycle et à des doses plus élevées.

Il n'est pas possible de comparer les bilans globaux, les femelles revenant en cstrus n'étant pas toujours inséminées. On peut estimer cependant, que I 40 à I $5^{\circ}$ veaux pour Ioo vaches vêlant à la suite du traitement doivent permettre d'obtenir 120 à I 25 veaux pour Ioo vaches traitées si les retours sont correctement détectés et si les animaux sont inséminés à nouveau et dans des conditions sanitaires plus favorables.

Les résultats portés dans le tableau 6 indiquent que le taux de mortinatalité croît avec la taille de la portée. Comparés avec les données d'autres auteurs (tabl. I 4) ces chiffres montrent un taux supérieur pour les naissances doubles et sensiblement inférieur pour les naissances triples et quadruples. De nombreux facteurs ont pu agir (poids des veaux, durée de gestation, suralimentation, influence du taureau...) 
TABIEAU I3

Taux de naissances gémellaires : revue bibliographique

\begin{tabular}{|c|c|c|c|c|}
\hline & Iraitement & $\begin{array}{l}\text { Nbre veaux nés } \\
\text { pour } 100 \mathrm{M} . \mathrm{B} . \\
\text { suite au traitement }\end{array}$ & $\begin{array}{l}\text { Nbre total veaux } \\
\text { nés pour } 100 \\
\text { vaches traitées }\end{array}$ & Observations \\
\hline GORDON et al, 1962 & PMSG & $1: 0$ & - & $\begin{array}{l}\text { Chiffre calculé sans tenir } \\
\text { compte des régulations } \\
\text { de nombre de foetus }\end{array}$ \\
\hline BELLOW' et al., 1969 & $\begin{array}{l}\text { Synchro } \\
\text { I'SH }\end{array}$ & $115^{*}$ & & $\begin{array}{l}\text { * Mises bas après } 2 \text { ser- } \\
\text { vices }\end{array}$ \\
\hline Mavleion et al., 1970 & PMSG & $\begin{array}{l}150 \\
140 \\
148\end{array}$ & $\begin{array}{l}122,4 \\
123,7 \\
131,1^{* *}\end{array}$ & $\begin{array}{l}\text { ** Chiffre calculé : retours } \\
\text { non inséminés }\end{array}$ \\
\hline TURMAN et al., 1971 & $\begin{array}{c}\text { PMSG } \\
\text { fractionnée }\end{array}$ & 192 & $13:$ & \\
\hline $\begin{array}{c}\text { Vincent et Mills, } \\
197:\end{array}$ & $\begin{array}{l}\text { Synchro } \\
\text { FSH }\end{array}$ & 126 & - & Retours non inséminés \\
\hline
\end{tabular}

TABLEAU I 4

Mortalité des veaux à la naissance en fonction de la taille de la portée

\begin{tabular}{|c|c|c|c|c|c|}
\hline \multirow[b]{2}{*}{ Références } & \multicolumn{5}{|c|}{ Taille de la portée } \\
\hline & 1 & 2 & 3 & ' & 5 \\
\hline \multicolumn{6}{|c|}{ I. - Naissances gémellaires provoquées } \\
\hline GORDON et al., 1962 & $3, \mathbf{t}$ & 10,0 & 54,2 & - & - \\
\hline TURMAN et al., $1971 \ldots$. & 3,5 & 0 & 511,0 & $6 ; 2,5$ & 60,0 \\
\hline Vincent et Mills, $1972 \ldots \ldots \ldots$ & 1,6 & 5,11 & - & 一 & - \\
\hline \multicolumn{6}{|c|}{ II. - Naissances gémellaires naturelles } \\
\hline JoHANSSON, 1932 .. & 5,1 & 17,5 & 一 & - & --- \\
\hline Richter, $1955 \ldots \ldots \ldots \ldots \ldots$ & 3,7 & 5,1 & -.. & $\ldots$ & $-\ldots$ \\
\hline DAERR et GRLNIRT, $1970 \mathrm{~b} \ldots \ldots$ & 25,5 & $6 !, 2$ & -- & - & - \\
\hline
\end{tabular}

et les effectifs sont faibles. De fait, les taux de mortalité en fonction de la taille de la portée observés dans des conditions de la pratique sans aucune surveillance particulière, calculés sur des effectifs plus importants, rejoignent ceux de la littérature (I. N. R. A., non publiés).

$\begin{array}{lll}\text { Doubles }(348) \ldots \ldots & \text { I } 8,3 \text { p. IOO } \\ \text { Triples (I I } 4) \ldots \ldots & 4 \mathbf{I}, 2 & - \\ \text { Quadruples }(48) \ldots . & 62,0 & -\end{array}$


On peut espérer les diminuer en améliorant la surveillance du vêlage. Quoi qu'il en soit, malgré une telle mortalité on conserve dans tous les cas $I, 6$ veaux vivants, soit un supplément de 0,65 veau par rapport au 0,95 restant après naissance simple.

Infuence du mâle sur la mortalité embryonnaire, le taux d'avortements, la prolificité et la mortalité des veaux.

Les données du tableau 5 montrent dans le lot I un effet du taureau utilisé pour l'insémination sur différents critères :

Les taux de conception sont équivalents, mais on constate que les 12 avortements sont tous liés à l'utilisation du taureau Cécil, dont 9 de portées multiples. A la mise bas, on obtient I29 p. Ioo de veaux nés avec le taureau Cécil contre I 88 p. Ioo avec le taureau Cervantès. Les deux taureaux utilisés pour le lot 2 ne semblent pas différents ; cette importance du choix du taureau utilisé pour les fécondations dans les expériences de naissances gémellaires ne semble pas avoir été observée auparavant. Selon d'autres sources d'informations, le taureau Cécil serait porteur d'une anomalie chromosomique (trans-location) pouvant être reliée à cette fertilité plus faible.

\section{II. - Incidence de la gémellité sur les performances des veaux}

Poids de naissance des veaux, durée de gestation.

De nombreux auteurs ont montré que la durée de gestation est plus courte et que le poids des veaux à la naissance est plus faible en cas de gestation multiple (voir HENDy et Bowman, I970 pour revue). Ceci a été signalé notamment dans toutes les expériences d'induction de naissances gémellaires (tabl. 15). Nous le retrouvons dans cette expérience.

\section{TABLEAU I5}

Poids de naissance des veaux. Durées de gestation

\begin{tabular}{|c|c|c|c|c|c|c|c|c|c|c|c|}
\hline \multirow{3}{*}{ Référence } & \multirow{3}{*}{ Race } & \multicolumn{5}{|c|}{ I)urée de gestation (jours) } & \multicolumn{4}{|c|}{ Poids de naissance } & \multirow[t]{2}{*}{$\left(k_{g}\right)$} \\
\hline & & \multicolumn{9}{|c|}{ Taille de la portée } & \\
\hline & & 1 & $\ddot{2}$ & $: 3$ & 't+ & 5 & 1 & 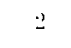 & 3 & 4 & 5 \\
\hline 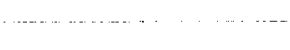 & 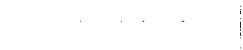 & - ....- & & . . & & & - & & & $\ldots$ & $\ldots$ \\
\hline GORDON et al., 1962 & Hereford & 282 & 273,2 & $26-2,1$ & - & 一 & 35,6 & 31,0 & 25,9 & 一 & 一 \\
\hline MaUleon et al., 1970 & $F F P X$ & 276,0 & 270,5 & 260,8 & - & - & 39,3 & 31,5 & 22,5 & - & - \\
\hline YURMAN et al., 1971 & Angus-Hereford & 280,8 & 277,4 & 269,2 & $26,2,5$ & 258,0 & 37,7 & $2 x, 1$ & 20,9 & 16,5 & $13,+i$ \\
\hline Vinclint et Mills, 1972 & $\begin{array}{c}\text { Croisés Angus } \\
\text { Brahman Charolais } \\
\text { Hereford }\end{array}$ & $98_{t}^{\prime}, 8$ & 281,1 & - & $-\cdots$ & - & $\mid 3 \cdot 3,1$ & 25,2 & - & : - & : - \\
\hline & & & & & & & & & & & \\
\hline
\end{tabular}

Mais on sait également que le génotype du fœetus modifie la durée de gestation. En particulier, l'insémination d'une femelle de race à courte durée de gestation $(F F P N=277 \mathrm{j})$ par de la semence de taureau de race à gestation longue (Charolais 
$=290 \mathrm{j}$; ou Blonde d'Aquitaine $=293$ j) entraîne un allongement de la gestation. Si les problèmes liés aux naissances gémellaires (poids des veaux, mortalité, rétentions placentaires...) sont liés au raccourcissement de la durée de gestation il peut être intéressant d'utiliser le croisement avec une race à durée de gestation longue. De plus, ces races à durée de gestation plus longue produisent en général des veaux à potentiel de croissance plus élevé. Telle était l'hypothèse conduisant à inséminer les femelles Frisonnes de ces deux expériences avec des taureaux Blonds d'Aquitaine. La comparaison des résultats du tableau 7 avec ceux de MAULEON et al. (I970) du tableau $5_{5}$ montre que effectivement, on obtient un allongement de la durée de gestation puisque les gestations doubles et triples des expériences rapportées ici sont d'une durée égale aux gestations simples de l'expérience de MaUleov et al. (1970) (femelles FFPN $\times$ mâle $F F P N$ ). De même, les poids de naissance des veaux doubles et triples sont augmentés.

Enfin, on note (tabl. 9) un effet favorable de la suralimentation en fin de gestation. Ceci a fait l'objet d'une étude par ailleurs (Bosc et Chupin, I975).

\section{Croissance des veaux.}

Les veaux du lot I (tabl. Io) ont subi un régime restreint avant l'entrée en station. Les poids obtenus par la suite ne correspondent pas à l'optimum. On peut constater cependant que les veaux nés multiples n'ont pas souffert de ce régime défavorable plus que les veaux simples.

Ces résultats, ainsi que ceux publiés par ailleurs (tabl. I6) permettent de dire que si les veaux multiples naissent plus légers, ils ont par la suite une croissance normale et atteignent des poids qui restent dans les limites de variations de ce que l'on peut observer pour des veaux simples. Seuls, Bellows et al. (1970), pratiquant un sevrage à 3 jours, indiquent une croissance plus élevée pour des doubles que pour les simples. En réalité, il s'agit de croissances assez faibles.

TABLEAU 16

Croissance des veaux en fonction de la taille de la portée

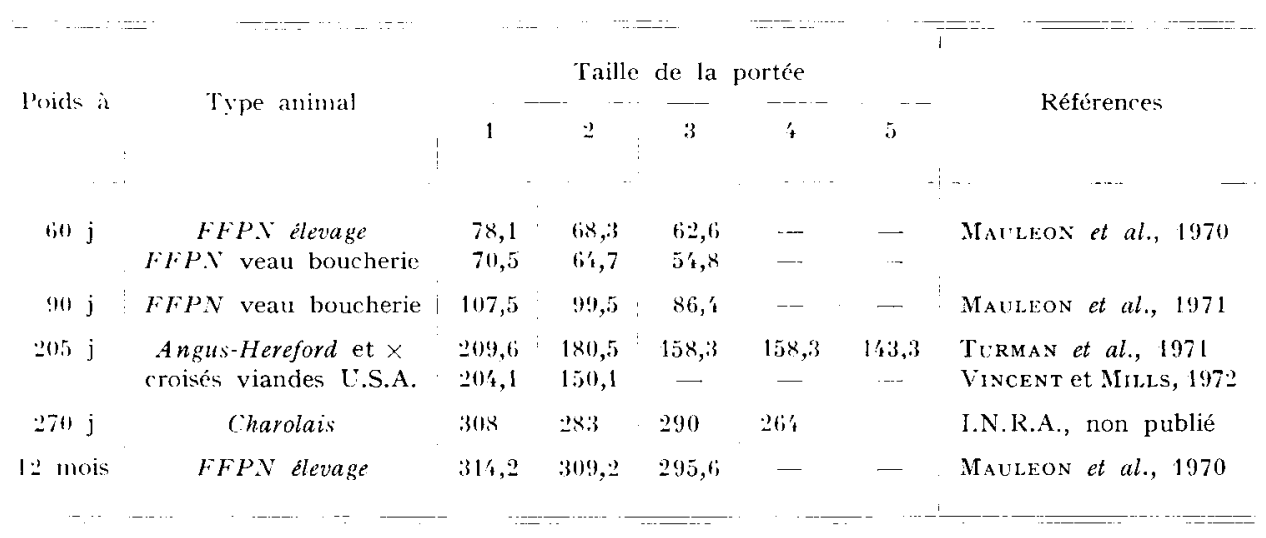




\section{III. - Incidence de l'induction hormonal' sur les performances des vaches}

Rétentions placentaires.

Il est bien connu que les naissances gémellaires sont associées arec une augmentation de la fréquence des rétentions placentaires comparées aux naissances simples: Gordon et al. (I962) (+ i5,2 p. IOO), DaERr et Grunert (I970 b) (+ 4I,9 p. IOO), Turmax et al. (I97I) ( + 4I,o p. IOO), Vincentr et Miris (I972) (+ 28,4 p. IOO).

Ces rétentions placentaires seraient liées au raccourcissement de la gestation. On sait (HAMmoND, I959) que la suralimentation en fin de gestation en diminue la fréquence. Ce fait a été confirmé avec les résultats de cette expérience rapportée précédemment (Bosc et Churs, 1975).

\section{Fertilité ultivieure.}

Morrow et al. (I966) indiquent que les difficultés de vêlages (y compris les rétentions placentaires) ont un effet sur la reprise de l'activité de la reproduction. Au contraire, WAGNER et al. (I97 I) OSINGA et al. (I97I) avec un traitement systéniatique à l'aide d'antibiotiques et LAUDERDALE (1972) sans aucun traitement ne notent pas d'effets négatifs des rétentions placentaires. Tous ces auteurs insistent sur la nécessité de ne pas pratiquer une délivrance manuelle forcée. Dans l'étude rapportée ici, nous n'avons pas observé de différences de fécondité des vaches l'année suivante (tabl. I I). Un traitement antibiotique sans extraction forcée du placenta a donc suffi à éviter les complications après rétentions placentaires.

Les données de la bibliographie lorsqu'aucun traitement systématique après la mise bas n'est effectué indiquent une fertilité plus faible après naissances gémellaires : Boyd et REED (I96I) cités par Hendy et Bowman (I970), DAERr et GrunerT (I970 a).

L’intervalle moyen entre vêlages serait long après naissances gémellaires (BowMax et Hendy, I970). Après induction expérimentale, Turmax et al. (I97 I) indiquent que 25 p. Ioo des vaches ayant vêlé de plusieurs veaux ne sont pas fécondées pendant la période d'allaitement mais le sont après le sevrage.

Finfin, il faut noter que GoRDor et al. $\left(\mathrm{Ig}^{62}\right)$ montrent une influence de l'alimentation pendant la gestation multiple sur la fertilité ultérieure. Le nombre de vaches suralimentées dans notre étude n'est pas suffisant pour permettre une étude de ce point.

\section{Production laitière.}

Les données rassemblées par Hendy et Bowmax (I970) sont contradictoires, certains auteurs constatent une baisse, d'autres une augmentation, d'autres enfin, aucun effet. Comme nous-mêmes, Bowmax et Hexdy (I970), MaUlEoxi et al. (I970), ne trouvent pas d'effet significatif. 


\title{
CONCLUSION
}

Les expériences rapportées ici montrent qu'il est possible d'induire dans les conditions pratiques des élevages privés une augmentation de la fréquence des naissances gémellaires. Si le taux de superovulation dépend principalement des paramètres du traitement hormonal, le taux de naissances gémellaires et les conséquences sur les performances des vaches ou des veaux dépendent de facteurs tout autres (état sanitaire, fertilité des reproducteurs utilisés, niveaux nutritionnels en fin de gestation, déroulement de la mise bas...). La réussite ou l'échec d'une induction hormonale de naissances gémellaires repose plus sur la maitrise de ces facteurs (qui conditionnent, bien que de façon moins tranchée, la productivité numérique d'un élevage bovin en conduite normale) que sur les paramètres du traitement hormonal lui-même.

Dans l'expérience présentée ici, nous arons notamment mis en évidence que certains taureaux utilisés normalement en insémination artificielle pouvaient se caractériser dans les conditions plus difficiles d'une gestation multiple par une mortalité embryonnaire et fortale élevée.

En éliminant ce facteur "taureaux", et malgré un état sanitaire défarorab] 132 élerages réagissent au test de détection de la brucellose sur 92), nous avons pu obtenir un maxinum de 129 veaux nés pour roo vaches mises en traitement.

Pour une extension de cette technique l'effort doit porter principalement sur la simplification des traitements, notamment par l'utilisation conjointe des traitements de synchronisation de l'oestrus et sur la sensibilisation des éleveurs à l'importance d'une intervention rapide à la mise bas.

$$
\text { Recu poun publicatorn en juin l9ij }
$$

\section{REMERCIEMENTS}

Nous remercions .I. Rondeau, directeur de la C. O. O. P. E. I. S. O., d'avoir permis la mise en place de ces expériences, MM. Aurejac, Boxnefoy et Delexis pour leur aide dans la réalisation des traitements et l'Tnstitut tcchnique de l'Elevage bovin qui a assuré le financement de l'expérimentation

\section{SUMMARY}

\author{
HORMONAI, INDUCTION OF MULTIPLE BIRTHS IN COWS. \\ MAIN EFFECTS ON THE, PERFORMANCES
}

The possibility of using PMSG in practice to enhance the multiple birth rate was studied on 256 lactating FFPN cows (French Frisian Black and White breed) belonging to farmers in the South West of France. The calf crop was reduced by the presence of brucellosis (45.7 p. Ioo of the herds) and by the fact that one of the bulls used for the artificial insemination exhibitecl fertility disorders. The gestation rates after one AI and for the overall campaign varied from

Annales de Zooterhnie. - I976. 
45.8 to 66.2 p. Ioo and from 88.3 to 94 . I p. Ioo, respectively. For Ioo cows, which calved after the treatment, the rate of calves born varied from I 8 to $188 \mathrm{p}$. Ioo. In the best group (high PMSG level, bull with normal fertility), I 29 calves were obtained from roo treated cows.

The calves from multiple births were lighter and born earlier (reduced gestation length) than those from single births, but these differences could be reduced by the choice of the bull used for the AI and by overfeeding of the cows during late gestation. Growth rates till the age of 300 days were not significantly different from those of the single-born calves.

No significant effect was observed either on the fertility of the cows after multiple births or on the milk production during or after multifoctal gestations.

\section{RÉFÉRENCES BIBLIOGRAPHIQUES}

Bellows R. A., Axdersox D. C., Short R. E., ig69. Dose-response relationships in synchronized beef heifers treated with follicle stimulating hormone. J. Anim. Sci., 28, 638-644.

Bellows R. A., Short R. E., Wiltbank N. H., Pahnish O. F., ig7o. Multiple births and artificial rearing of calves. J. Anim. Sci., 30, rozo (Abstr.).

13ellows R. A., ShorT R. E., 1972. Superovulation and nultiple births in beef cattle. Ith Biennal Symposium on Animal Reproduction, 34, suppl. I, 67-69.

13ose M. J., Chupin D., r975. Jissai d'application de l'électrocardiographie au diagnostic de gestation uni ou multifotale chez la Vache. Ann. Zootech., 24, i I 7-1 23.

Bowman J.C., Hendy C. R. C., Ig7o. The incidence, repeatability and effect on dam performance of twinning in British Friesian cattle. Anim. Prod., 12, 55-62.

BOYD H., REED H.C. B., I96I. Investigations into the incidence and causes of infertility in dairy cattle. I. lertility variations. II. Influence of some management factors affecting the semen and insemination conditions. III. Influence of kale feeding, milk production and management factors associated with farming intensity. Br. Vet. J., 117, 8-35, 74-86, I 29-200 (cités par HENDY et Bowman, 1970).

1)AERr H.C., Grunert J., I970a. Die Zwillingsträchtigkeit bein Rind in geburtshilflicher und gesundheitlicher sicht. Dtsch. Tierärztl. Wschr., 77, I-24.

DAERR H.C., GRUNERT E., I970 b. Gesundheit und Fruchtbarkeit von deutschen schwarzbunten Rindern nach erschwerter Zwillingsgeburt. Dtsch. Tieräztl. If schr., gry, $201-204$.

Gorvon I., Williams G., EDwards J., ig62. The use of serum gonadotrophin (PMS) in the induction of twin pregnancy in the cow. J. Agric. Sci., 59, I43-198.

Hafez E.S. E., Rajakoski E., Anderson P. B., Frost O. L., Smith G., Ig64. Problems of gonadotropin induced multiple pregnancy in beef cattle. Amer. J. Fet. Res., 25, Io7.

Hammond J., I959. Twinning in cattle for beef production, I'et. Kec., 71, r68-r69.

Hendi C. R. C., Bowman J. C., r97o. Twinning in cattle. Anim. Breed. Abstr., 38, 22-37.

Johansson I., I932. The sex ratio and multiple births in cattle. Z. Ziucht. Reihe B, 24, I83-268 (cite par HENDY et Bowman, 1970).

Laster D. B., Turman E. J., Stephens D. F., Revbarger R. E., ig7i. Ovulation rates of becf cows and heifers treated with equine gonadotropin (PMS) and chorionic gonadotropin (HCG). J.Anim. Sci., 33, $443-449$.

Lauderdale J. W., 1972. Eiffect of corticoid administration on bovine pregnancy. J. Am. I'et. Med. Ass., $160,867-871$.

Mauleon P., Bosc M., Courot M., Pelot J., Schneberglir J., Ortavant R., ig7o. Obtention de naissances gemellaires après superovulation limitée et caractéristiques zootechniques des vaches productrices et des veaux jumeaux. Ann. Biol. anim. Bioch. Biophys., no Hors-série, Fr, II3-122.

Osinga A., Stegenga T., Jochle W., I97I. Initiation of parturition in dairy cows with a synthetic corticoid. Zucht., 6, 64-69.

Richter F., r95.5. Untersuchungen Über der verbleib der Nachkommen von RL-Kühen des Höhenvich. Z. Tierzuicht. ZuichtBiol., 65, 223-242 (cité par HENDY et Bowman, 1970).

Schwartz I. L., Shelby I), R., I969. Induced multiple ovulation in the bovine. J. Anim. Sci., 29, I 98 (Abstr.).

Turman E. J., Laster D. B., Renbarger R. E., Sthphevs D. J., I97i. Multiple births in beef cows treated with equine gonadotropin (PMS) and chorionic gonadotropin (HCG). J.Anim.Sci., 32, $962-967$.

Vincent C. K., Minls A. C., I972. Gonadotropin levels for multiple births in beef cattle. J. Anim. Sci., 34, 77-8I.

Wagner W. C., William R. L., Evans L. E., I97I. Induced parturition in the beef cow. J. Anim. Sci., 33, 1964 (Abstr.). 